\title{
Dietary Sodium/Potassium Intake Does Not Affect Cognitive Function or Brain Imaging Indices
}

\author{
Kristen L. Nowak ${ }^{a}$ Linda Fried $^{\text {b, c }}$ Anna Jovanovich ${ }^{a, d}$ Joachim Ix \\ Kristine Yaffe ${ }^{f}$ Zhiying You ${ }^{a}$ Michel Chonchol $^{a}$ \\ ${ }^{a}$ Division of Renal Diseases and Hypertension, University of Colorado Denver Anschutz Medical Campus, Aurora, \\ CO, USA; ${ }^{b}$ Division of Renal-Electrolyte, University of Pittsburgh, Pittsburgh, PA, USA; ${ }^{c}$ Renal Section, Veterans \\ Affairs Pittsburgh Healthcare System, Pittsburgh, PA, USA; ${ }^{d}$ Renal Section, Medical Service, Denver Veterans Affairs \\ Medical Center, Denver, CO, USA; ' Division of Nephrology, University of California San Diego, San Diego, CA, USA; \\ ${ }^{f}$ Departments of Psychiatry, Neurology, and Epidemiology, University of California San Francisco, San Francisco, \\ CA, USA
}

\section{Keywords}

Ageing $\cdot$ Cognitive dysfunction · Epidemiology $\cdot$ Nutrition · Potassium · Risk factors · Sodium

\begin{abstract}
Background: Dietary sodium may influence cognitive function through its effects on cerebrovascular function and cerebral blood flow. Methods: The aim of this study was to evaluate the association of dietary sodium intake with cognitive decline in community-dwelling older adults. We also evaluated the associations of dietary potassium and sodium:potassium intake with cognitive decline, and associations of these nutrients with micro- and macro-structural brain magnetic resonance imaging (MRI) indices. In all, 1,194 participants in the Health Aging and Body Composition study with measurements of dietary sodium intake (food fre-
\end{abstract}

(c) 2018 S. Karger AG, Basel

E-Mail karger@karger.com www.karger.com/ajn quency questionnaire [FFQ]) and change in the modified Mini Mental State Exam (3MS) were included. Results: The age of participants was $74 \pm 3$ years with a mean dietary sodium intake of $2,677 \pm 1,060 \mathrm{mg} /$ day. During follow-up $(6.9 \pm$ 0.1 years), 340 (28\%) had a clinically significant decline in $3 \mathrm{MS}$ score ( $\geq 1.5 \mathrm{SD}$ of mean decline). After adjustment, dietary sodium intake was not associated with odds of cognitive decline (OR $0.96,95 \% \mathrm{Cl} 0.50-1.84$ per doubling of sodium). Similarly, potassium was not associated with cognitive decline; however, higher sodium:potassium intake was associated with increased odds of cognitive decline (OR 2.02 [95\% Cl 1.01-4.03] per unit increase). Neither sodium or potassium alone nor sodium:potassium were associated with micro- or macro-structural brain MRI indices. These results are limited by the use of FFQ. Conclusions: In communitydwelling older adults, higher sodium:potassium, but not sodium or potassium intake alone, was associated with decline
Kristen L. Nowak, PhD, MPH

Division of Renal Diseases and Hypertension:

12700 E 19th Ave C281

Aurora, CO 80045 (USA)

E-Mail Kristen.Nowak@ucdenver.edu 
in cognitive function, with no associations observed with micro- and macro-structural brain MRI indices. These findings do not support reduction dietary sodium/increased potassium intake to prevent cognitive decline with aging.

(C) 2018 S. Karger AG, Basel

\section{Introduction}

With advancing age, subtle impairments in cognition are common even in the absence of clinically apparent dementia [1]. Conceptual reasoning, memory, processing speed, attention, and working memory tend to gradually decline over time, and there is also a decline in both gray matter and white matter volume in the brain $[1,2]$. Older adults with mild cognitive impairment have an increased likelihood of experiencing continued cognitive decline and to progress to dementia [3].

Most older adults exceed recommended levels of dietary sodium intake [4]. The adverse consequences of high dietary sodium intake upon blood pressure are well appreciated [5], with additional evidence for increased risk of cardiovascular events [6]. Dietary sodium intake may also influence cognitive function through its effects upon cerebrovascular function and cerebral blood flow. High dietary sodium intake is associated with impaired vascular endothelial function [7], which is associated with cerebral small vessel disease [8], a major risk factor for cognitive impairment with advancing age [9]. However, the relation between dietary sodium intake and cognitive dysfunction is not well understood. Additionally, little is known about the association of dietary potassium intake and cognitive impairment, and the interaction of dietary sodium and potassium intake may better predict risk than either nutrient alone [10], as high dietary potassium may counter the adverse effects of high dietary sodium [11].

Accordingly, the aim of this study was to evaluate the association of dietary sodium intake with cognitive decline over time in community-dwelling older adults. We hypothesized that greater sodium intake would be independently associated with cognitive decline. Given important interactions between dietary potassium and sodium in modulating cardiovascular risk [12], we also evaluated the associations of dietary sodium intake and the ratio of dietary sodium to potassium intake with cognitive decline. Finally, to provide mechanistic insight, we evaluated the association of sodium intake with microand macro-structural brain magnetic resonance imaging (MRI) indices in participants of this substudy.

\section{Materials and Methods}

\section{Study Population}

The study methods of the Health, Aging and Body Composition (Health $\mathrm{ABC}$ ) study have been described previously [13]. In all, 3,075 participants 70-79 years of age were recruited from a sample of Medicare beneficiaries residing in areas surrounding Pittsburgh, PA and Memphis, TN between April 1997 and June 1998. Eligible participants reported no difficulties walking $1 / 4$ mile, climbing ten steps, or performing basic activities of daily living; they were all free from life-threatening illness and planned to remain in the geographic area for at least 3 years.

For the present analysis, year 3 cognitive testing (Modified Mini-Mental State Examination [3MS]) was defined as baseline measurements, as this was the first assessment following dietary data collected in year 2. Participants were excluded if they had missing food frequency questionnaire (FFQ) data $(n=362)$ or had values for dietary data outside the range defined of physiologically plausible (total kcals $<800 \mathrm{kcal}$ for males or $<600 \mathrm{kcal}$ for females [14], sodium intake $<500 \mathrm{mg} /$ day, and/or potassium intake $<1,000$ $\mathrm{mg}$ /day; $n=64$ ), leaving $n=2,649$ with dietary data. An additional $n=1,205$ were excluded due to missing data on incident cognitive impairment (3MS score at years 3 and 10) and/or due to prevalent cognitive impairment at baseline ( $n=263$; 3 MS score $\geq 1.5$ $\mathrm{SD}$ below the mean). Finally, 250 remaining participants were excluded due to missing covariates (described below), leaving 1,194 participants in the current analysis. All participants provided written informed consent, and this study was approved by and conducted in accordance with the ethical standards of the investigational review boards at the participating centers, as well as in accordance will the Declaration of Helsinki.

\section{Study Variables}

Dietary sodium intake was the primary predictor variable, with dietary potassium intake and the ratio of sodium to potassium intake (sodium:potassium) evaluated as secondary predictor variables. Dietary sodium intake, as well as dietary potassium and total caloric intake, were assessed at baseline (year 2) by a 108-item interviewer-administered FFQ (Block Dietary Data Systems, Berkley, CA, USA), with a food list developed specifically for the Health ABC study [14]. Cognitive function was evaluated at baseline (year 3 ) by the $3 \mathrm{MS}$, a general cognitive battery with higher scores indicating better cognitive function [15]. Incident cognitive impairment was defined as a decline in $3 \mathrm{MS}$ score $\geq 1.5 \mathrm{SD}$ ) above the mean decline in completion score (mean \pm SD change in $3 \mathrm{MS}$ score was $-2.8 \pm 8.6$ ).

Confounders related to dietary sodium intake and cognitive impairment were selected a priori as potential covariates. Race, sex, education (year 1), and smoking status (year 3) were determined by an interviewer-administered questionnaire at baseline. Education was classified into 3 categories (less than high school, high school graduate, or post-secondary). Smoking was defined as current, former, or never. Body mass index (BMI) was calculated using body weight measured using a calibrated balance beam scale and height measured using a stadiometer (year 2). Age at year 2 was defined as baseline age. Blood pressure was measured in a rested, seated position (year 2). Cardiovascular disease (CVD) was defined as a history of congestive heart failure or myocardial infarction by combining participant self-report with appropriate medication use and 5 years prior of Centers for Medicare and
58

Am J Nephrol 2018;47:57-65 DOI: $10.1159 / 000486580$
Nowak/Fried/Jovanovich/Ix/Yaffe/You/ Chonchol 
Medicaid data (year 1). Hypertension and diabetes status were determined by similar methods (year 2). Estimated glomerular filtration rate was calculated using the Chronic Kidney Disease Epidemiology Collaboration equation [16]. Creatinine was measured using the isotope dilution mass spectrometry traceable methodology (year 3).

\section{Healthy Brain Substudy}

A subset of Health $\mathrm{ABC}$ participants were included in the Healthy Brain substudy, which began in year 10 and included measurements of brain macro- and micro-structures by MRI. Of the 1,194 participants included in the main analyses, 248 had MRI data available for mechanistic analysis regarding the association of dietary sodium intake with micro- and macro-structural brain MRI indices in year 10. The details of the Healthy Brain substudy and MRI acquisition have been described previously [17]. MRI scanning was performed using a 3T Siemens Tim Trio MR scanner at the MR Research Center of the University of Pittsburgh using a Siemens 12-channel head coil. Macro-structure white matter hyperintensities (WMH) volume was obtained from T2-weighted FLAIR image using an automated method for quantification and localization of WMH. WMH quantification was done using a fuzzy connected algorithm and total WMH volume was estimated by summing all the voxels classified as WMH and normalized for brain volume. Total gray matter volume (GMV) was estimated in cubic millimeters by summing all voxels classified as this tissue type. Fractional anisotropy (FA), a marker of white matter integrity, was measured as restricted to normal appearing white matter. Cerebral atrophy index was calculated as intracranial volume - parenchyma volume, where parenchyma volume $=\mathrm{GMV}+$ white matter volume from total brain. Correction factors were used for clearer presentation of data (WMH multiplied by 1,000, GMV, white matter volume, and intracranial volume divided by 1,000 , cerebral atrophy divided by 10 ) [17].

\section{Statistical Analyses}

The association of dietary sodium intake with incident cognitive impairment was assessed using logistic regression models. Dietary sodium intake was evaluated by quartiles, using quartile 1 predefined in the analysis to serve as the reference group due to being the lowest intake. We also examined dietary sodium as a continuous predictor variable (per every doubling of sodium intake $\left[\log _{2}\right.$ transform $]$ ). The initial model was unadjusted and then multivariable adjusted models were performed to include age, sex, and race (model 1), model 1 plus education, smoking status, $\mathrm{BMI}$, systolic blood pressure (SBP), estimated glomerular filtration rate, and history of hypertension, CVD, and diabetes (model 2), and model 2 plus dietary potassium and total caloric intake (model 3). We also considered restricted cubic regression spline analysis; however, results showed no evidence of a nonlinear association with spline analysis; thus, this nonlinear analysis was determined not to be appropriate. We tested for an interaction of dietary sodium with both sex and race, and found no significant interactions $(p>0.73)$; thus, stratified analyses were not performed. Similarly, there was no significant interaction of sex or race with either potassium or sodium:potassium intake $(p>$ $0.24)$.

As secondary analyses, dietary potassium intake and sodium:potassium were considered predictor variables using the models described above (substituting dietary sodium for potassium in model 3 when dietary potassium was the predictor variable, and eliminating dietary sodium in model 3 when sodium:potassium was the predictor variable). As an additional secondary analysis, linear regression was used to assess the association of baseline dietary sodium, potassium, and sodium:potassium intake with change in $3 \mathrm{MS}$ score at year 10. Last, linear regression models were used in the Healthy Brain substudy to examine the association of dietary sodium intake with WMH volume, GMV, FA of white matter, and cerebral atrophy index, with the same covariates described above. Quartiles were redefined based on this sub-cohort. The natural log was used for skewed variables (WMH volume).

As an additional secondary analysis, we considered the associations of dietary sodium, potassium, and sodium:potassium intake on cognitive function in a sub-group of participants who had baseline comorbidities of history of hypertension, diabetes, and/or CVD $(n=372)$. Finally, we added diuretic use (self-report [18]) to a final model evaluating the associations of dietary sodium, potassium, and sodium:potassium intake on cognitive function.

Measures of cognitive function and covariates at baseline were summarized by dietary sodium quartiles (as well as by potassium and sodium:potassium quartiles) and presented as mean (SD) or median (interquartile range [IQR]) for continuous variables and $\%$ for categorical variables. $3 \mathrm{MS}$ score was square root transformed and $\mathrm{WMH}$ volume was log-transformed in analyses to achieve a normal distribution. Comparisons across quartiles were made using a chi-square test for categorical data and analysis of variance for continuous variables. Two-tailed values of $p<0.05$ were considered statistically significant for all analyses. All statistical analyses were performed using SAS version 9.4.

\section{Results}

\section{Participant Characteristics at Baseline}

A total number of 1,194 participants were included in the main analysis, with a mean follow-up interval of $6.9 \pm$ 0.1 years. Among these participants, the mean \pm SD age was $74 \pm 3$ years and $71.4 \%(n=853)$ were White. The mean dietary sodium intake was $2,677 \pm 1,060 \mathrm{mg} /$ day (Table 1). The median (IQR) baseline 3MS Examination score was 95 (90-97), with the lowest score occurring in the highest quartiles of sodium intake. Individuals with higher dietary sodium intake were less likely to be female, more likely to have CVD, and tended to have a higher BMI. Total caloric intake, dietary potassium intake, and dietary sodium:potassium were also greater in those with higher sodium intake (Table 1).

\section{Relation between Dietary Sodium Intake and Incident Cognitive Impairment}

The incidence of cognitive impairment was 10.1, 9.0, 8.1 , and $13.7 \%$ for quartile 1 (597-1,921 mg/day), quartile 2 (1,921-2,511 mg/day), quartile 3 (2,517-3,264 mg/ day), and quartile 4 (3,264-11,565 $\mathrm{mg}$ /day) respectively 
Table 1. Baseline characteristics of study participants by quartiles of baseline dietary sodium intake by food frequency questionnaire

\begin{tabular}{|c|c|c|c|c|c|}
\hline Variable & $\begin{array}{l}\text { Q1 } \\
(597-1,921 \\
\text { mg/day; } n=298)\end{array}$ & $\begin{array}{l}\text { Q2 } \\
(1,921-2,511 \\
\text { mg/day; } n=299)\end{array}$ & $\begin{array}{l}\text { Q3 } \\
(2,511-3,264 \\
\text { mg/day; } n=297)\end{array}$ & $\begin{array}{l}\text { Q4 } \\
(3,264-11,565 \\
\mathrm{mg} / \text { day; } n=300)\end{array}$ & $p$ value \\
\hline Race (White), \% & 72.8 & 71.0 & 75.8 & 66.0 & 0.06 \\
\hline Gender, female, \% & 69.5 & 61.9 & 50.2 & 35.7 & $<0.0001$ \\
\hline Education, \% & & & & & 0.44 \\
\hline Post-secondary & 51.3 & 53.5 & 55.6 & 50.3 & \\
\hline Smoking, $n(\%)$ & & & & & 0.99 \\
\hline Never smoker & 50.0 & 50.2 & 49.8 & 48.0 & \\
\hline Current smoker & 4.4 & 4.7 & 3.7 & 4.0 & \\
\hline Former smoker & 45.6 & 45.2 & 46.5 & 48.0 & \\
\hline CVD, $\%$ & 8.7 & 7.0 & 7.4 & 14.3 & 0.04 \\
\hline Diabetes, \% & 8.7 & 9.0 & 11.8 & 13.7 & 0.16 \\
\hline CKD-EPI eGFR, $\mathrm{mL} / \mathrm{min} / 1.73 \mathrm{~m}^{2}$ & $78 \pm 15$ & $78 \pm 15$ & $77 \pm 14$ & $79 \pm 16$ & 0.73 \\
\hline Diuretic use, $\%$ & 26.2 & 20.9 & 22.7 & 22.6 & 0.12 \\
\hline Total, kcals/day & $1,201 \pm 274$ & $1,566 \pm 267$ & $1,975 \pm 324$ & $2,704 \pm 741$ & $<0.0001$ \\
\hline Dietary potassium, mg/day & $2,159 \pm 631$ & $2,577 \pm 688$ & $3,081 \pm 724$ & $3,959 \pm 1,187$ & $<0.0001$ \\
\hline Dietary sodium, mg/day & $1,546 \pm 275$ & $2,195 \pm 173$ & $2,849 \pm 210$ & $4,113 \pm 853$ & $<0.0001$ \\
\hline Dietary sodium:potassium, ratio & $0.8 \pm 0.2$ & $0.9 \pm 0.3$ & $1.0 \pm 0.2$ & $1.1 \pm 0.3$ & $<0.0001$ \\
\hline 3MS, score & $95(90-97)$ & $95(90-97)$ & $95(91-97)$ & $94(88-97)$ & 0.005 \\
\hline
\end{tabular}

Data are mean $\pm \mathrm{SD}$, median (interquartile range) or $\%$.

BMI, body mass index; SBP, systolic blood pressure; DBP, diastolic blood pressure; CVD, cardiovascular disease; CKD-EPI eGFR, estimated glomerular filtration rate using the chronic kidney disease EPI equation; 3MS, modified mini mental status examination.

Table 2. Associations (OR [95\% CI]) between quartiles of baseline dietary sodium intake and incident cognitive impairment by logistic regression models

\begin{tabular}{|c|c|c|c|c|c|}
\hline Model & $\begin{array}{l}\text { Q1 } \\
(597-1,921 \\
\text { mg/day; } n=298)\end{array}$ & $\begin{array}{l}\text { Q2 } \\
(1,921-2,511 \\
\text { mg/day; } n=299)\end{array}$ & $\begin{array}{l}\text { Q3 } \\
(2,511-3,264 \\
\text { mg/day; } n=297)\end{array}$ & $\begin{array}{l}\text { Q4 } \\
(3,264-11,565 \\
\text { mg/day; } n=300)\end{array}$ & $\begin{array}{l}\text { Continuous (per } \\
\text { doubling of } \\
\text { sodium intake) }\end{array}$ \\
\hline Model 1 & Ref. & $0.85(0.49-1.49)$ & $0.77(0.43-1.36)$ & $1.21(0.71-2.04)$ & $1.22(0.86-1.73)$ \\
\hline Model 2 & Ref. & $0.86(0.49-1.53)$ & $0.74(0.41-1.33)$ & $1.15(0.67-1.97)$ & $1.14(0.80-1.63)$ \\
\hline Model 3 & Ref. & $0.82(0.45-1.47)$ & $0.65(0.34-1.27)$ & $0.94(0.42-2.10)$ & $0.96(0.50-1.84)$ \\
\hline
\end{tabular}

Model 1: adjusted for age, sex, and race. Model 2: adjusted for model 1 + education, smoking, BMI, SBP, history of hypertension, stroke, CVD, and diabetes, and estimated glomerular filtration rate. Model 3 is further adjusted for total kcals and dietary potassium intake. Continuous is for every doubling of sodium intake ( $\log _{2}$ transformation).

$(p=0.12)$. In both unadjusted and adjusted analyses, increased dietary sodium intake was not associated with increased odds of incident cognitive impairment compared to the reference group (quartile 1; Table 2). In the fully adjusted model, the odds of cognitive impairment in the highest quartile was 0.94 (95\% CI 0.42-2.10) compared to the reference group. Similarly, for every doubling of dietary sodium intake, the odds of incident cognitive impairment was 0.96 (95\% CI 0.50-1.84). Addition of diuretic use did not change this association
60 
Table 3. Associations (OR [95\% CI]) between quartiles of dietary potassium and sodium:potassium intake and incident cognitive impairment by logistic regression models

\begin{tabular}{llllll}
\hline Potassium model & $\begin{array}{l}\text { Q1 }(1,029-2,200 \\
\text { mg/day; } n=298)\end{array}$ & $\begin{array}{l}\text { Q2 (2,200-2,765 } \\
\text { mg/day; } n=298)\end{array}$ & $\begin{array}{l}\text { Q3 (2,765-3,521 } \\
\text { mg/day; } n=299)\end{array}$ & $\begin{array}{l}\text { Q4 (3,521-12,179 } \\
\text { mg/day; } n=299)\end{array}$ & $\begin{array}{l}\text { Continuous (per doubling } \\
\text { of potassium intake) }\end{array}$ \\
\hline Unadjusted & Ref. & $0.75(0.44-1.27)$ & $0.90(0.54-1.50)$ & $0.78(0.46-1.31)$ & $0.86(0.60-1.25)$ \\
Model 1 & Ref. & $0.81(0.47-1.38)$ & $1.00(0.60-1.70)$ & $0.81(0.47-1.38)$ & $0.90(0.63-1.31)$ \\
Model 2 & Ref. & $0.79(0.57-1.68)$ & $0.98(0.57-1.68)$ & $0.83(0.48-1.44)$ & $0.92(0.62-1.35)$ \\
Model 3 & Ref. & $0.70(0.39-1.23)$ & $0.77(0.42-1.39)$ & $0.51(0.24-1.10)$ & $0.55(0.30-1.01)$ \\
\hline Sodium:potassium & Q1 (0.350-0.743; & Q2 (0.743-0.904; & Q3 (0.904-1.083; & Q4 (1.083-2.400; & Continuous (per unit \\
model & $n=298)$ & $n=297)$ & $n=299)$ & $n=299)$ & increase in Na+/K+) \\
\hline Unadjusted & Ref. & $1.09(0.62-1.93)$ & $1.00(0.56-1.78)$ & $1.94(1.15-3.25)$ & $3.46(1.84-6.49)$ \\
Model 1 & Ref. & $1.16(0.65-2.07)$ & $0.90(0.50-1.63)$ & $1.61(0.94-2.74)$ & $2.53(1.31-4.88)$ \\
Model 2 & Ref. & $1.19(0.66-2.15)$ & $0.88(0.48-1.62)$ & $1.43(0.82-2.48)$ & $2.09(1.05-4.13)$ \\
Model 3 & Ref. & $1.16(0.64-1.55)$ & $0.84(0.45-1.55)$ & $1.36(0.77-2.38)$ & $2.02(1.01-4.03)$ \\
\hline
\end{tabular}

Model 1: adjusted for age, sex, and race. Model 2: adjusted for model $1+$ education, smoking, BMI, SBP, history of hypertension, stroke, CVD, and diabetes, and estimated glomerular filtration rate. Model 3 is further adjusted for total kcals (and dietary sodium intake; potassium model only). Continuous is for every doubling of potassium intake $\left(\log _{2}\right.$ transformation) and per unit increase in sodium:potassium intake.

(model 3 highest quartile OR 0.94 [95\% CI 0.42-2.10] vs. reference group). Similarly, there was no association between quartiles of dietary sodium intake or dietary sodium considered a continuous variable and change in 3MS score at year 10 (online suppl. Table 1; for all online suppl. material, see www.karger.com/ doi/10.1159/000486580). Results were very similar when a definition of cognitive impairment as a decline in $3 \mathrm{MS}$ score $\geq 1.0 \mathrm{SD}$ above the mean decline in completion score was applied (not shown). Finally, in the subgroup of participants with baseline comorbidities of hypertension, diabetes, and/or CVD, there remained no association between dietary sodium intake and odds of incident cognitive impairment, similar to the overall cohort (model 3 highest quartile OR 1.57 [95\% CI 0.36-6.79] compared to the reference group).

\section{Relation between Dietary Sodium Intake and Brain MRI Indices}

The baseline characteristics of the 248 participants in the Healthy Brain substudy are shown in online supplemental Table 2. Similar to the main analysis, individuals with higher sodium intake were less likely to belong to the female gender and had greater total caloric intake, dietary potassium intake, and dietary sodium:potassium intake. Cerebral atrophy index was also greater in individuals with greater dietary sodium intake. However, in the fully adjusted models, dietary sodium intake was not signifi- cantly associated with any of the measured brain MRI indices (WMH, GMV, FA of white matter, and cerebral atrophy index; online suppl. Table 3).

\section{Secondary Analyses}

To provide additional insight due to known interactions between dietary sodium and potassium, baseline dietary potassium intake and dietary sodium:potassium intake were considered predictor variables in secondary analyses. Baseline characteristics of participants according to quartiles of baseline dietary potassium intake are presented in online supplemental Table 4. Individuals with higher dietary potassium intake were more likely to be White, less likely to be female, and had higher total caloric intake and dietary sodium intake, with lower dietary sodium:potassium intake. Hypertension was less likely in those with both the lowest and highest dietary potassium intake. 3MS score was the highest in those with the greatest dietary potassium intake.

Similar to dietary sodium intake, whether potassium intake was considered a categorical or continuous variable, there was no association of dietary potassium intake with incident cognitive impairment in any model (Table 3 ). Addition of diuretic use did not change this association (model 3 highest quartile OR 0.55 [95\% CI 0.561.17] vs. reference group). Results were similar in linear regression analysis considering the change in 3MS score at year 10 a continuous outcome (online suppl. Table 5). 
In the sub-group of participants with baseline comorbidities of hypertension, diabetes, and/or CVD, there was also no association of dietary potassium intake with incident cognitive impairment (model 3 highest quartile OR 0.32 [95\% CI 0.07-1.46] vs. reference group). Dietary potassium intake was not associated with brain MRI parameters (not shown).

Baseline characteristics of study participants according to quartiles of baseline dietary sodium:potassium intake are shown in online supplemental Table 6. Individuals with the highest ratio were less likely to be White, less likely to be female, less likely to have post-secondary education, and had higher total caloric intake. There was also a tendency toward higher SBP and greater prevalence of CVD. Baseline 3MS was lower in individuals with higher dietary sodium:potassium intake, and diuretic use was lower in individuals with lower dietary sodium:potassium intake.

The incidence of cognitive impairment was 8.4, 9.1, 8.1 , and $17.8 \%$ for quartile $1(0.350-0.743)$, quartile 2 (0.743-0.904), quartile $3(0.904-1.083)$, and quartile 4 $(1.083-2.400)$ respectively $(p=0.02)$. In the fully adjusted model, for every unit increase in dietary sodium:potassium intake, there was a 2.02 times greater odds (95\% CI 1.01-4.03) of cognitive impairment; however, there were no significant differences across quartiles compared to the reference group (Table 3 ). Addition of diuretic use did not significantly change this association (model 3 highest quartile OR 1.35 [95\% CI 0.76-2.39] vs. reference group; per unit increase in dietary sodium:potassium intake OR 2.03 [95\% CI 1.01-4.11]). There were also no significant associations in linear regression analysis considering the change in $3 \mathrm{MS}$ score at year 10 a continuous outcome (online suppl. Table 7). Results were similar in the subgroup of participants with baseline comorbidities of hypertension, diabetes, and/or CVD (OR 3.69 [95\% CI 0.9814.0] per unit increase in dietary sodium:potassium intake; no significant difference across quartiles compared to the reference group). Dietary sodium:potassium intake was not associated with brain MRI parameters (not shown).

\section{Discussion}

Contrary to our hypothesis, we found no association between greater dietary sodium intake and cognitive decline in community-dwelling older adults. Similarly, we observed no independent association between dietary potassium intake and cognitive decline. However, higher dietary sodium:potassium intake was associated with slightly increased odds of cognitive decline, highlighting the importance of interactions between these 2 nutrients. Finally, in participants of the Healthy Brain substudy, we found no associations of either nutrient or their ratio with micro- or macro-structural brain MRI indices.

An association between dietary sodium intake and cognitive function has been suggested previously in both humans and animal models [19-25]. In contrast to our findings, sodium intake measured by FFQ was independently associated with greater decline in $3 \mathrm{MS}$ score over a 3-year period in older adults (the Nutrition and Successful Aging [NuAge] study); however, this association was observed only in sedentary individuals [19]. The lack of classification of sedentary behavior in Health $\mathrm{ABC}$, as well as longer follow-up period may account for differences between these studies. In contrast, in a prospective study of older women, while hypertension was a risk factor for the development of mild cognitive impairment over the 9 year follow-up period, there was no effect modification according to sodium intake by FFQ [21]. Results in cross-sectional analyses are conflicting, with evidence of an association between 24-h urinary sodium excretion and worse cognitive performance in patients with newly diagnosed hypertension [20], as well as worse cognitive performance with lower sodium intake in community-dwelling middle-aged older adults [22].

Several rodent models have suggested that increased sodium over a 4-12-week period intake may impair cognitive function, including learning and both short- and long-term memory [23-25]. Despite lack of association observed in the current study, these mechanistic animal studies suggest a relationship between sodium intake and cognition may exist. Hypertonic saline delivery directly into the cerebral ventricle increases blood pressure and sympathetic tone, thus high dietary sodium may trigger a higher blood pressure set point and promote hypertension [26]. Hypertension is a known risk factor for the deterioration of cognitive function [27]. However, cognitive impairment can also occur in rodents independent of changes in blood pressure [23], and in the NuAge study, the association of dietary sodium intake with cognitive function was independent of hypertension [19]. High dietary sodium intake also increases hippocampal reactive oxygen species production and decreases antioxidant enzyme expression [23-25], in addition to disturbing synaptic plasticity in the hippocampus [25]. The
Nowak/Fried/Jovanovich/Ix/Yaffe/You/ Chonchol 
effects of high sodium may also be mediated via vascular function, as sodium can induce vascular endothelial dysfunction [7], which plays a pathogenic role in the development of cerebral small vessel disease [8]. Perhaps due to reduced cerebral blood flow, vascular endothelial dysfunction is also a major risk factor for cognitive impairment, $[9,28]$.

Additionally, dietary potassium intake has considerable importance in modulating the effects of dietary sodium, and intake is low in industrialized nations, leading to a high sodium to potassium ratio [29]. High potassium intake may counter adverse effects of high sodium intake and attenuate the adverse cardiovascular consequence of high dietary sodium intake, $[11,12]$ and the ratio of sodium to potassium may provide greater insight regarding cardiovascular risk than either nutrient alone [10]. Much less is known about the influence of dietary potassium intake on cognitive function. Findings in previous epidemiological studies have been conflicting [30,31]. In a longitudinal study (17-year follow-up) in community-dwelling older adults, higher dietary potassium intake (by FFQ) was associated with reduced risk of incident vascular dementia [28]. This association was possibly mediated via lower blood pressure, as altered potassium concentrations in cerebrospinal fluid can modulate blood pressure [32]. Notably, the analysis was not adjusted for dietary sodium intake.

Conversely, a later analysis found that higher dietary potassium intake (also by FFQ) was associated with increased risk of developing mild cognitive impairment 8 years later [31]. However, in a rodent model of Alzheimer's disease, increased potassium intake attenuated histopathological markers of disease and improved cognitive function, which was associated with decreased markers of oxidative stress and inflammation in the hippocampus and cortex, as well as improved longterm potentiation [32]. In the current study, we found no independent association of dietary potassium intake with cognitive decline; however, the risk of cognitive decline was increased with increasing dietary sodium:potassium intake. However, this association was observed only in the continuous but not categorical analysis, and not in the linear regression analysis of change in 3MS score; thus, this finding should be interpreted cautiously. To our knowledge, the association of sodium:potassium and cognitive function has not been examined previously.

To provide additional mechanistic insight regarding the associations of sodium and potassium with cognitive function, we evaluated the associations of these nutrients with brain macro- and micro-structures by MRI in participants in the Healthy Brain substudy. Cerebral white matter lesions increase with age and are associated with impaired cognitive function [33]. They are believed to represent microvascular disease resulting from chronic hypoperfusion [33]. On the other hand, GMV of the medial temporal area was greater for those who maintained cognitive function over time in the Healthy Brain substudy [17]. Decreased FA in white matter is indicative of microstructural abnormalities such as demyelination and axonal loss and is sensitive as early markers of cognitive impairment and performance decline [34]. We found no significant associations of dietary sodium, potassium, or sodium:potassium intake with any of these indices, or with cerebral atrophy index. However, these findings are limited by the fact that these assessments were performed in a relatively small number of participants in the Healthy brain substudy.

The major strength of this study is the consideration of both sodium and potassium, as well as their ratio, as predictors of cognitive decline with aging, as these associations have not been examined previously. Additionally, we utilized a well-characterized cohort with comprehensive information about potential covariates being available, and longitudinal follow-up over approximately 7 years. However, there are also several limitations to this analysis. Sodium intake was measured using the FFQ rather than 24-h urinary sodium excretion, and the former tends to underestimate sodium intake [35]. As discussed in a recent study evaluating the association of sodium intake with mortality and incident CVD in the Health ABC study, the mean sodium intake in this population was relatively low, and this may have made it more difficult to ascertain an association [36]. Additionally, the predictor variables were measured just once and dietary habits could have changed over the follow-up period. Related, there was a temporal separation between assessment of diet and the brain MRI parameters, and the sample size for the brain MRI indices was quite limited. Our assessment of cognitive function was also limited; however, the $3 \mathrm{MS}$ score is considered a measure of global cognitive function, including orientation, attention, immediate and short-term recall, and language [15]. Finally, the Health ABC participants were communitydwelling individuals with good functional capacity; thus, these results may not reflect the general aging population.

In conclusion, in a population of community-dwelling older adults, neither dietary sodium nor potassium intake was associated with cognitive decline or with brain mac- 
ro- and micro-indices by MRI. There is possibly a role for the interaction between sodium and potassium (i.e., their ratio) and cognitive decline; however, this association should be investigated further in a larger population. Additional longitudinal analyses measuring 24 -h urinary sodium and potassium excretion levels are also indicated, including populations of chronic kidney disease patients. It would be of particular interest to study brain MRI indices in a larger sample size as well. The current results do not support reduced dietary sodium or increased potassium intake as an intervention to prevent cognitive decline with aging.

\section{Disclosure Statement}

The authors have no conflicts of interest to disclose.

\section{Funding Sources}

This research was supported by the National Institutes of Health (NIH) National Institute on Aging (NIA; contracts N01AG-6-2101; N01-AG-6-2103; N01-AG-6-2106; grants R01AG028050; R01-AG029232) and NIH National Institute of Nursing Research (NINR; grant R01-NR012459). Kristen Nowak is supported by NIH National Institute of Diabetes and Digestive and Kidney Disease (NIDDK) K01-DK103678.

\section{References}

1 Harada CN, Natelson Love MC, Triebel KL: Normal cognitive aging. Clin Geriatr Med 2013;29:737-752.

2 Institute of Medicine: Cognitive Aging: Progress in Understanding and Opportunities for Action. Washington, The National Academies Press, 2015, Vol. 1, pp 1-330.

3 Flicker C, Ferris SH, Reisberg B: Mild cognitive impairment in the elderly: predictors of dementia. Neurology 1991;41:1006-1009.

4 Centers for Disease Control and Prevention (CDC): Usual Sodium Intakes Compared with Current Dietary Guidelines - United States, 2005-2008. MMWR Morb Mortal Wkly Rep 2011;60:1413-1417.

5 Institute of Medicine: Sodium Intake in Populations: Assessment of Evidence. Washington, The National Academies Press, 2013, vol $1, \mathrm{pp} 1-224$.

6 O'Donnell MJ, Yusuf S, Mente A, Gao P, Mann JF, Teo K, McQueen M, Sleight P, Sharma AM, Dans A, Probstfield J, Schmieder RE: Urinary sodium and potassium excretion and risk of cardiovascular events. JAMA 2011; 306:2229-2238.

7 Tzemos N, Lim PO, Wong S, Struthers AD, MacDonaldTM: Adversecardiovascular effects of acute salt loading in young normotensive individuals. Hypertension 2008;51:1525-1530.

8 Markus HS: Genes, endothelial function and cerebral small vessel disease in man. Exp Physiol 2008;93:121-127.

9 Prins ND, van Dijk EJ, den Heijer T, Vermeer SE, Jolles J, Koudstaal PJ, Hofman A, Breteler MM: Cerebral small-vessel disease and decline in information processing speed, executive function and memory. Brain 2005;128(pt 9):2034-2041.

10 Cook NR, Obarzanek E, Cutler JA, Buring JE, Rexrode KM, Kumanyika SK, Appel LJ, Whelton PK; Trials of Hypertension Prevention Collaborative Research Group: Joint effects of sodium and potassium intake on subsequent cardiovascular disease: the trials of hypertension prevention follow-up study. Arch Intern Med 2009;169:32-40.
11 Bussemaker E, Hillebrand U, Hausberg M, Pavenstadt H, Oberleithner H: Pathogenesis of hypertension: interactions among sodium, potassium, and aldosterone. Am J Kidney Dis 2010;55:1111-1120.

12 Aaron KJ, Sanders PW: Role of dietary salt and potassium intake in cardiovascular health and disease: a review of the evidence. Mayo Clin Proc 2013;88:987-995.

13 Cesari M, Penninx BW, Newman AB, Kritchevsky SB, Nicklas BJ, Sutton-Tyrrell K, Rubin SM, Ding J, Simonsick EM, Harris TB, Pahor M: Inflammatory markers and onset of cardiovascular events: results from the health ABC study. Circulation 2003;108:2317-2322.

14 Houston DK, Nicklas BJ, Ding J, Harris TB, Tylavsky FA, Newman AB, Lee JS, Sahyoun NR, Visser M, Kritchevsky SB; Health ABC Study: Dietary protein intake is associated with lean mass change in older, communitydwelling adults: the health, aging, and body composition (Health ABC) study. Am J Clin Nutr 2008;87:150-155.

15 Teng EL, Chui HC: The modified mini-mental state (3MS) examination. J Clin Psychiatry 1987;48:314-318

16 Levey AS, Stevens LA, Schmid CH, Zhang YL, Castro AF 3rd, Feldman HI, Kusek JW, Eggers P, Van Lente F, Greene T, Coresh J; CKDEPI (Chronic Kidney Disease Epidemiology Collaboration): A new equation to estimate glomerular filtration rate. Ann Intern Med 2009;150:604-612.

17 Rosano C, Aizenstein HJ, Newman AB, Venkatraman V, Harris T, Ding J, Satterfield S, Yaffe K; Health ABC Study: Neuroimaging differences between older adults with maintained versus declining cognition over a 10 year period. Neuroimage 2012;62:307-313.

18 Cesari M, Penninx BW, Newman AB, Kritchevsky SB, Nicklas BJ, Sutton-Tyrrell K, Tracy RP, Rubin SM, Harris TB, Pahor M: Inflammatory markers and cardiovascular disease (the Health, Aging and Body Composition [Health $\mathrm{ABC}$ ] study). Am J Cardiol 2003; 92:522-528.
19 Fiocco AJ, Shatenstein B, Ferland G, Payette H, Belleville S, Kergoat MJ, Morais JA, Greenwood CE: Sodium intake and physical activity impact cognitive maintenance in older adults: the NuAge study. Neurobiol Aging 2012;33: 829 e821-e828.

20 Afsar B: The relationship between cognitive function, depressive behaviour and sleep quality with 24 -h urinary sodium excretion in patients with essential hypertension. High Blood Press Cardiovasc Prev 2013;20:19-24.

21 Haring B, Wu C, Coker LH, Seth A, Snetselaar L, Manson JE, Rossouw JE, WassertheilSmoller S: Hypertension, dietary sodium, and cognitive decline: results from the women's health initiative memory study. Am J Hypertens 2016;29:202-216.

22 Rush TM, Kritz-Silverstein D, Laughlin GA, Fung TT, Barrett-Connor E, McEvoy LK: Association between dietary sodium intake and cognitive function in older adults. J Nutr Health Aging 2017;21:276-283.

23 Liu YZ, Chen JK, Li ZP, Zhao T, Ni M, Li DJ, Jiang CL, Shen FM: High-salt diet enhances hippocampal oxidative stress and cognitive impairment in mice. Neurobiol Learn Mem 2014;114:10-15.

24 Chugh G, Asghar M, Patki G, Bohat R, Jafri F, Allam F, Dao AT, Mowrey C, Alkadhi K, Salim S: A high-salt diet further impairs age-associated declines in cognitive, behavioral, and cardiovascular functions in male Fischer brown Norway rats. J Nutr 2013;143:1406-1413.

25 Ge Q, Wang Z, Wu Y, Huo Q, Qian Z, Tian Z, Ren W, Zhang X, Han J: High salt diet impairs memory-related synaptic plasticity via increased oxidative stress and suppressed synaptic protein expression. Mol Nutr Food Res 2017;61.

26 Takahashi H, Yoshika M, Komiyama Y, Nishimura M: The central mechanism underlying hypertension: a review of the roles of sodium ions, epithelial sodium channels, the renin-angiotensin-aldosterone system, oxidative stress and endogenous digitalis in the brain. Hypertens Res 2011;34:1147-1160. 
27 Carnevale D, Mascio G, D’Andrea I, Fardella V, Bell RD, Branchi I, Pallante F, Zlokovic B, Yan SS, Lembo G: Hypertension induces brain $\beta$-amyloid accumulation, cognitive impairment, and memory deterioration through activation of receptor for advanced glycation end products in brain vasculature. Hypertension 2012;60:188-197.

28 Andresen J, Shafi NI, Bryan RM Jr: Endothelial influences on cerebrovascular tone. J Appl Physiol (1985) 2006;100:318-327.

29 Adrogue HJ, Madias NE: Sodium and potassium in the pathogenesis of hypertension. $\mathrm{N}$ Engl J Med 2007;356:1966-1978.

30 Ozawa M, Ninomiya T, Ohara T, Hirakawa Y, Doi Y, Hata J, Uchida K, Shirota T, Kitazono T, Kiyohara Y: Self-reported dietary intake of potassium, calcium, and magnesium and risk of dementia in the Japanese: the hisayama study. J Am Geriatr Soc 2012;60:15151520.

31 Cherbuin N, Kumar R, Sachdev PS, Anstey $\mathrm{KJ}$ : Dietary mineral intake and risk of mild cognitive impairment: the path through life project. Front Aging Neurosci 2014;6:4.

32 Cisternas P, Lindsay CB, Salazar P, Silva-Alvarez $C$, Retamales $R M$, Serrano FG, Vio $C P$, Inestrosa NC: The increased potassium intake improves cognitive performance and attenuates histopathological markers in a model of Alzheimer's disease. Biochim Biophys Acta 2015; 1852:2630-2644.

33 Kuo HK, Lipsitz LA: Cerebral white matter changes and geriatric syndromes: is there a link? J Gerontol A Biol Sci Med Sci 2004;59: 818-826.
34 Bozzali M, Cherubini A: Diffusion tensor MRI to investigate dementias: a brief review. Magn Reson Imaging 2007;25:969977.

35 Day N, McKeown N, Wong M, Welch A, Bingham S: Epidemiological assessment of diet: a comparison of a 7-day diary with a food frequency questionnaire using urinary markers of nitrogen, potassium and sodium. Int J Epidemiol 2001;30:309-317.

36 Kalogeropoulos AP, Georgiopoulou VV, Murphy RA, Newman AB, Bauer DC, Harris TB, Yang Z, Applegate WB, Kritchevsky SB: Dietary sodium content, mortality, and risk for cardiovascular events in older adults: the health, aging, and body composition (health ABC) study. JAMA Intern Med 2015;175: 410-419. 